\title{
Inflammatory Myofibroblastic Tumor of the Liver: A Diagnostic Challenge
}

\author{
Gülsüm Özlem Elpek \\ Department of Pathology, Akdeniz University Medical School, Antalya, Turkey
}

\begin{abstract}
Inflammatory myofibroblastic tumor (IMT) is an uncommon myofibroblastic neoplasm that was formerly included within the broad category of inflammatory pseudotumor (IPT). IMT is rarely encountered in the liver. Similar to IMT of other organs, the interchangeable use of the terms IMT and IPT in liver has made the analysis of these lesions difficult. In this review, clinical and pathological features of IMT of the liver are reviewed and the differential diagnosis of IMT is discussed, with emphasis on IPT and the other entities included in this large category. IMT can mimic malignant tumors. There are no known unique diagnostic clinical, laboratory, or radiological features. The definitive diagnosis of IMT depends on careful pathological examination. The histopathological evaluation of hepatic IMT reveals that, the myxoid/vascular pattern is the most frequently observed, followed by, in decreasing frequency, fibrous histiocytomalike pattern and hypocellular fibrous pattern. In IMT of the liver, anaplastic lymphoma kinase (ALK) expression reliably predicts the presence of an ALK gene rearrangement. The diagnosis of hepatic IMT depends on the dominant histopathological pattern, and the management of the disease is still controversial. IMT of the liver is a distinctive neoplasm of intermediate biological potential, and should be distinguished from the variety of lesions that are included under the broad category of IPT. Therefore, to avoid confusion regarding the true incidence and behavior of hepatic IMT, the term IPT should not be used interchangeably with IMT. The rarity of IMT in liver should not minimize its consideration in the differential diagnosis of liver tumors, especially in patients with tumor markers in normal range.

(C) 2014 The Second Affiliated Hospital of Chongqing Medical University. Published by XIA \& HE Publishing Ltd. All rights reserved.
\end{abstract}

Keywords: Inflammatory myofibroblastic tumor; Liver; Anaplastic lymphoma kinase; Inflammatory pseudotumor.

Abbreviations: AFP, alpha-fetoprotein; ALK, anaplastic lymphoma kinase; CEA, carcinoembryonic antigen; CFT, calcifying fibrous tumor; ESR, erythrocyte sedimentation rate; FH-IPT, fibrohistiocytic-type IPT; GIST, gastrointestinal stromal tumor; IMT, inflammatory myofibroblastic tumor; IPT, inflammatory psuedotumor; LP-IPT, lymphoplasmacytic-type IPT; RANB2, RAN binding protein 2; SFT, solitary fibrous tumor.

Received: 29 October 2013; Revised: 9 December 2013; Accepted: 10 December 2013

DOI of original article: 10.14218/JCTH.2013.00023.

Correspondence to: Gülsüm Özlem Elpek, Akdeniz University Medical School, Department of Pathology, Antalya 07070, Turkey. Email: elpek@akdeniz.edu.tr

\section{Introduction}

Inflammatory myofibroblastic tumors (IMTs) are uncommon neoplasms, and reports of them are increasing in the literature. These lesions can be found in various tissues and organs in the body. IMTs were formerly included within the broad category of non-neoplastic fibroinflammatory and neoplastic lesions referred to as inflammatory pseudotumor (IPT) ${ }^{1-3}$ However, over the last two decades, several studies have demonstrated distinctive, clinical, pathological, and molecular features relative to IPTs. ${ }^{1-3}$ At present IMTs are classified as a neoplasm of intermediate biological potential due to a tendency of recurrence, and the rare incidence of distant metastasis. ${ }^{4}$ Despite this classification in the current World Health Organization Histological Typing of Soft Tissue Tumors, ${ }^{4}$ the terms IMT and IPT have been used interchangeably in the past.

Since the first description of hepatic IPT by Pack and Baker in $1953,{ }^{5}$ over 300 cases have been reported. Until recently IMT of the liver fell within the broader definition of IPT. In this review, clinical and pathological features of both IPT and IMT of the liver are reviewed; in addition, the differential diagnosis of IMT will be discussed, with emphasis on IPT and the other entities included in this large category.

\section{Clinical features}

Published data suggest that recognition of IPT of the liver is increasing in frequency in the Far East. ${ }^{6}$ In a study of resected focal lesions in 403 patients, the incidence of hepatic IPT was reported to be $0.7 \% .{ }^{7}$ IPT presents in men in their $30 \mathrm{~s}$ and $40 \mathrm{~s}$, and is frequently associated with symptoms such as fever, abdominal pain, jaundice, and weight loss. ${ }^{8}$ On the other hand, hepatic IMT is very rare, and the real incidence is not known. Recently, Tang et al. ${ }^{9}$ analysed 64 cases diagnosed as IMT of the liver and observed that they had similar age and gender distributions as IPT. The most common clinical presentation was abdominal pain followed by fever and no symptoms. ${ }^{9,10}$ This is in contrast to IMT in other anatomical locations that are more common in children and adolescents with a slight female preponderance. ${ }^{11}$

Both in IPT and IMT of the liver, laboratory investigations often suggest an inflammatory process: leukocytosis, neutrophilia, elevated erythrocyte sedimentation rate (ESR) and $\mathrm{C}$-reactive protein (CRP), anemia, thrombocytosis, polyclonal hypergammaglobulinemia, and slightly elevated liver enzymes. ${ }^{8-10}$ Tumor markers such as serum alpha-fetoprotein (AFP) and carcinoembryonic antigen (CEA) were always normal, although elevated cancer antigen 19-9 (CA 19-9) was raised in some patients. Ogawa et al. proposed that IPT 


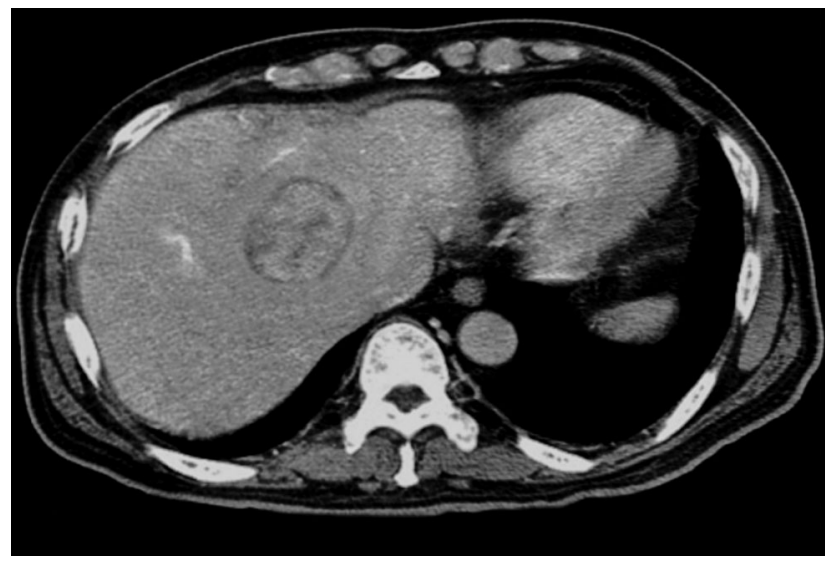

Fig. 1. A contrast enhanced CT scan discloses relatively well unencapsulated IMT on the right lobe of the liver.

itself could produce CA $19-9 .{ }^{12}$ Since the increase of this marker is also observed in patients with benign diseases of the hepatobiliary tract, it has been suggested that the increase in CA19-9 is not suitable for the diagnosis of IPT. ${ }^{13}$ While AFP levels are valuable in the discrimination of both conditions from malignant epithelial tumors, they cannot completely rule out malignancies where AFP levels may be normal such as fibro-lamellar hepatocellular carcinoma. ${ }^{13}$ In addition, radiological findings of these lesions are not specific, and their discrimination is not always possible ${ }^{8-10}$ (Fig. 1).

Although multiple IPTs or IMTs of the liver are described in some reports, ${ }^{14,21,31-33}$ they are mostly solitary lesions. ${ }^{8,9}$ Both IPT and IMT of the liver are more likely to involve the right lobe of the liver, with rare cases reported in the Spiegel lobe and hilar region. ${ }^{8-10,14-16}$ Although the exact etiology of IPT of the liver is unclear, infective agents have been considered, including actinomycosis, nocardia, and bacterial abcesses. ${ }^{17-19}$ More recently, the role of the biliary steroid lithocholic acid to the formation of IPT in the liver has been proposed. ${ }^{20}$ The etiology of hepatic IMT is not completely clear, although the demonstration of cytogenetic anomalies support a neoplastic origin. ${ }^{1-3,11}$ The definitive diagnosis of IMT rests on pathological evaluation since there are at present no known unique diagnostic clinical, laboratory or radiological features.

\section{Pathological features}

Grossly, both IPT and IMT have variegated appearances, and are generally unencapsulated. They typically have firm, fleshy, or gelatinous cut surfaces that rarely show hemorrhage, necrosis and calcification (Fig. 2).

Regardless of the organs of origin, the histolopathology of IMT of the liver is similar. Three basic histological patterns are often seen in combination within the same tumor: a myxoid/ vascular pattern, a compact spindle cell pattern, and a hypocellular fibrous pattern. ${ }^{1}$ The myxoid/vascular pattern has a fasciitis-like appearance, with loosely arranged plump spindle cells in a myxoid stroma and a prominent vasculature. The inflammatory infiltrate in these areas often contains fewer plasma cells than the other two patterns. The compact spindle cell pattern is characterized by a cellular proliferation of spindle cells with fascicular or storiform architecture in a collagenous stroma resembling fibrous histiocytoma.
Elpek GÖ: Inflammatory myofibroblastic tumor of the liver

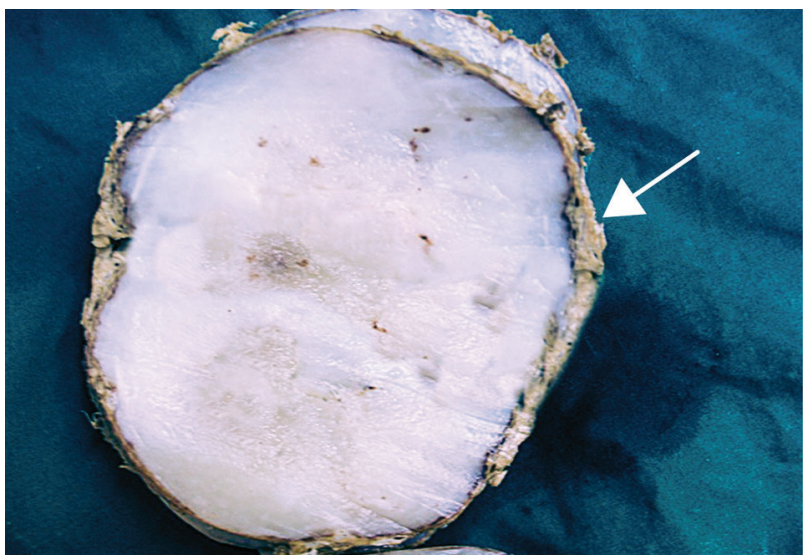

Fig. 2. Gross appearance of IMT. The tumor is circumscribed, white-tan in color on the whorled cut surface and surrounded by a narrow rim of liver parenchyma (arrow).

Numerous plasma cells and lymphocytes are present. The fibromatosis-like pattern is hypocellular, with elongated spindle cells, collagenous stroma, and scattered lymphoctyes. In IMT of the liver, the myxoid/vascular pattern is frequently observed followed by, in decreasing frequency, the fibrous histiocytoma like pattern and the hypocellular fibrous pattern (Fig. 3a-c). ${ }^{9}$

The spindle cells of IMT are typically uniform. Mild nuclear pleomorphism may be seen, but hyperchromasia is absent. Although, mitotic activity is generally low, and atypical mitoses are rare, ${ }^{1,21,23}$ IMTs may histologically evolve into a morphologically higher grade lesion with increased cellularity, marked nuclear atypia, frequent mitoses, atypical mitotic figures, and/ or necrosis. ${ }^{1,22,23}$ The cytological features of the morphologically higher grade tumors are variable, and include hypercellular spindle cell, epithelioid/histiocytoid, or

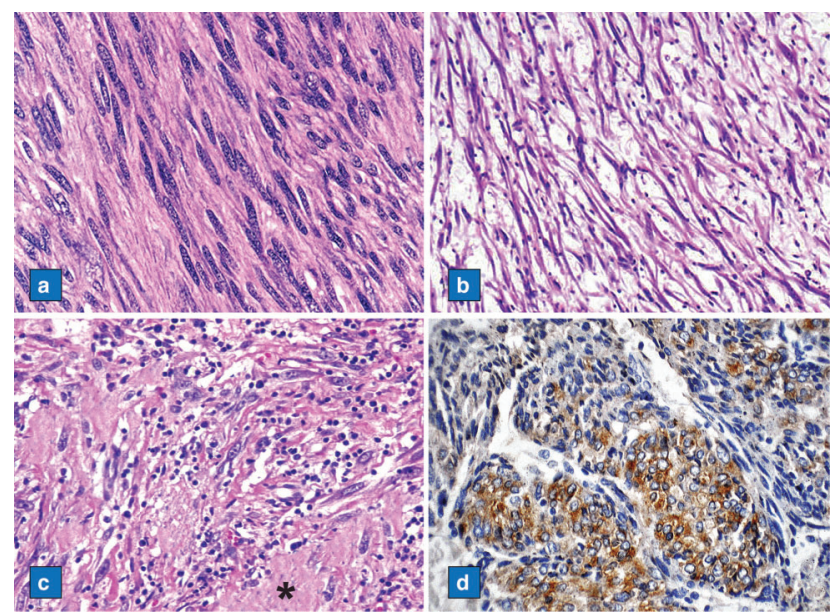

Fig. 3. Histpathological findings of IMT. a: Cellular areas in fibromatosis-like pattern composed of elongated spindle cells with vesicular nuclei in a collagenous background $(H \& E, x 400)$. b: Loosely arranged spindle cells in a myxoid background with scattered lymphocytes in myxoid-vascular pattern (H\&E, x100). c: The compact spindle pattern with spindle cells arranged in a fascicular pattern in a collagenous stroma (asterisk). Numerous lymphocytes and plasma cells are present. (H\&E, x200). d: Cytoplasmic staining of ALK in IMT of the liver (CD246, ALK protein counterstained with hematoxylin, $\times 200$ ) 
round cell morphology. ${ }^{1,22,23}$ There has been only one such case reported in the liver, a 34 year old man with hepatic IMT with round cell morphology with an unfavorable outcome. ${ }^{24}$

\section{Molecular features}

Rearrangements involving the anaplastic lymphoma kinase (ALK) locus on chromosome 2p23 have been documented for both pulmonary and extrapulmonary IMTs, providing further evidence for the neoplastic nature of these lesions and their distinction from IPT. 3,25 By immunohistochemical analysis, approximately 50 to $60 \%$ of IMTs are positive for ALK. ${ }^{1}$ A positive test is more commonly observed in younger patients but is not restricted to this population..$^{3,21,26,27}$ It is now known that ALK expression in IMT reliably predicts the presence of an ALK gene rearrangement. ${ }^{2,3,28}$ Recently ALK expression was reported in a few IMTs of the liver, including one recent case from our institution (Fig. 3d). 3,24,27,29,30-33

In IMT, localization of ALK within the cell appears to be determined by its fusion partner, and the pattern of ALK immunostaining may correlate with the specific gene fusion. ${ }^{2}$ Although a possible relationship between gene fusion type and prognosis has not been shown, cases with the RAN binding protein 2 (RANBP2)-ALK fusion demonstrated round cell transformation, suggesting that this subset may be more aggressive. ${ }^{2,34}$ RANBP2-ALK fusion was present in only one case of hepatic IMT with an aggressive course. ${ }^{24}$ However, at present, it is not possible to draw a conclusion about this finding, and more cases are required to verify this possibility.

\section{Differential diagnosis}

Differential diagnosis of hepatic IMT relies on the dominant histopathological pattern. The compact spindle cell pattern of hepatic IMT must be differentiated from metastatic sarcomatoid carcinoma, spindle cell sarcoma, and spindle cell melanoma. However, most of these tumors show at least focally prominent nuclear hyperchromasia, atypical mitosis, or necrosis. Moreover, plasma cells are not a prominent component of these tumors. ${ }^{11}$ Gastrointestinal stromal tumor (GIST) may also be considered in the differential diagnosis because, similar to IMT, an inflammatory infiltrate may be seen in GIST. However, it is patchy and plasma cells are not frequent. In contrast to IMT, GIST is consistently positive for c-kit (CD117) immunostaining. ${ }^{2,35}$ In some cases, differentiation of IMT with compact spindle cell pattern from follicular dendritic cell tumor/sarcoma may be difficult. In the latter tumors, the absence of plasma cells in the evenly distributed inflammatory infiltrate and the presence of CD21, CD23, and CD35 are useful findings to discriminate them from IMT. ${ }^{11}$ In the differential diagnosis of the fibromatosis-like or hypocellular pattern of hepatic IMT, solitary fibrous tumors (SFT) and calcifying fibrous tumors (CFT) must be considered. SFTs are positive for CD34, which is consistently negative in IMT. On the other hand, while cellularity in IMT is variable, CFT is uniformly hypocellular, with scattered dystrophic calcifications. ${ }^{36}$

Reactive processes dominated by granulation tissue may mimic the myxoid-vascular pattern of hepatic IMT. ${ }^{11}$ An organized vascular pattern, necrosis, and prominent inflammation in the adjacent tissue are important histological clues to a reactive process.

\section{Hepatic IPT versus IMT}

IPTs are characterized by the proliferation of fibroblasts or myofibroblasts and inflammatory cell infiltration composed of lymphocytes and plasma cells (Fig. $4 a-c$ ). In a recent study, Zen et al. ${ }^{37}$ classified hepatic IPT into two types: fibrohistiocytic-type and lymphoplasmacytic type. While, fibrohistiocytic-type IPT (FH-IPT) was associated with xanthogranulomatous inflammation and multinucleated giant cells, the lymphoplasmacytic-type IPT (LP-IPT) was histologically characterized by diffuse inflammatory cell infiltration, mainly by lymphocytes and plasma cells. They also demonstrated that the two types differed not only in pathological features but also in clinical features, location, and shape. FH-IPTs were located mainly in the liver parenchyma with mass-forming characteristics. In contrast, LP-IPTs showed periductal infiltrating-type hilar lesions. Patients with FH-IPT had subjective symptoms (fever, abdominal pain etc.), whereas patients with LP-IPT had liver dysfunction as determined by routine laboratory testing. According to the presence of diffuse IgG4 positive plasma cells in LP-IPTs, it has been suggested that this type could belong to IgG4related disease. In a more recent study, Yamamoto et al. ${ }^{32}$ compared the histopathological features with an emphasis on IgG4 expression between IMTs and IgG4-related diseases. They observed that IMTs were distinct from IgG4-related diseases by their ALK expression, low levels of IgG4 positive cell infiltration, and lack of obstructive phlebitis. Moreover, inflammatory infiltrate and fibrosis were not prominent in the peri-tumoral tissue adjacent to IMT. IgG4 likely plays no important role in the pathogenesis of IMT. Because the therapeutic strategy would be different in IMT and IgG4related diseases, careful pathological examination is necessary to discriminate between these two entities (Fig. $4 \mathrm{~d}$ and Fig. 5).

\section{Prognosis and management}

IMTs have been classified as tumors of intermediate biological potential because of their tendency for local recurrence and

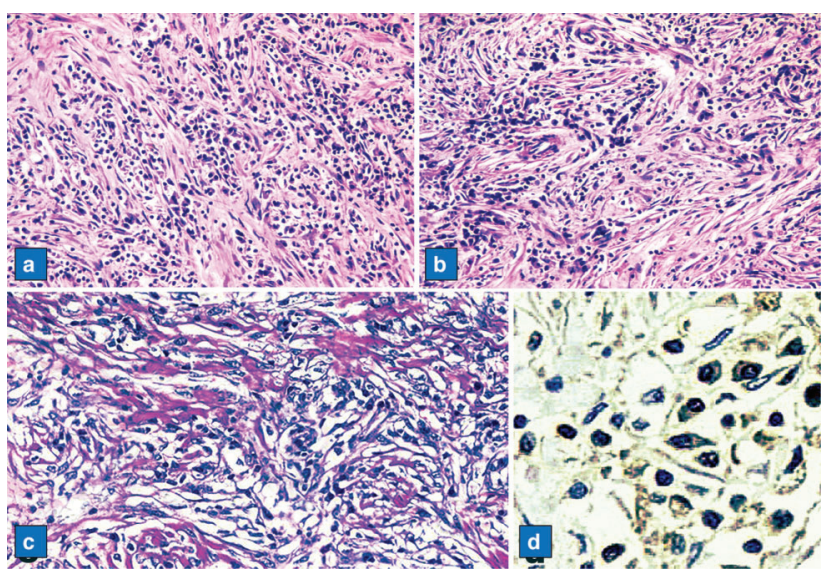

Fig. 4. Histpathological findings of IPT. a: IPT composed of diffuse infiltration of lymphocytes and plasma cells in a background of collagen (H\&E, x100). b: In some areas, lymphocytes and plasma cells are prominent around vascular structures (H\&E, $\times 100)$. c: In another case of IPT, spindle cells intermingled with lymphocytes, plasma cells, and histiocytes in a background of collagen deposits ( $x$ 200). d: IgG4 positive plasma cells in IPT presented in a and b ( $x 400)$ 
Elpek GÖ: Inflammatory myofibroblastic tumor of the liver
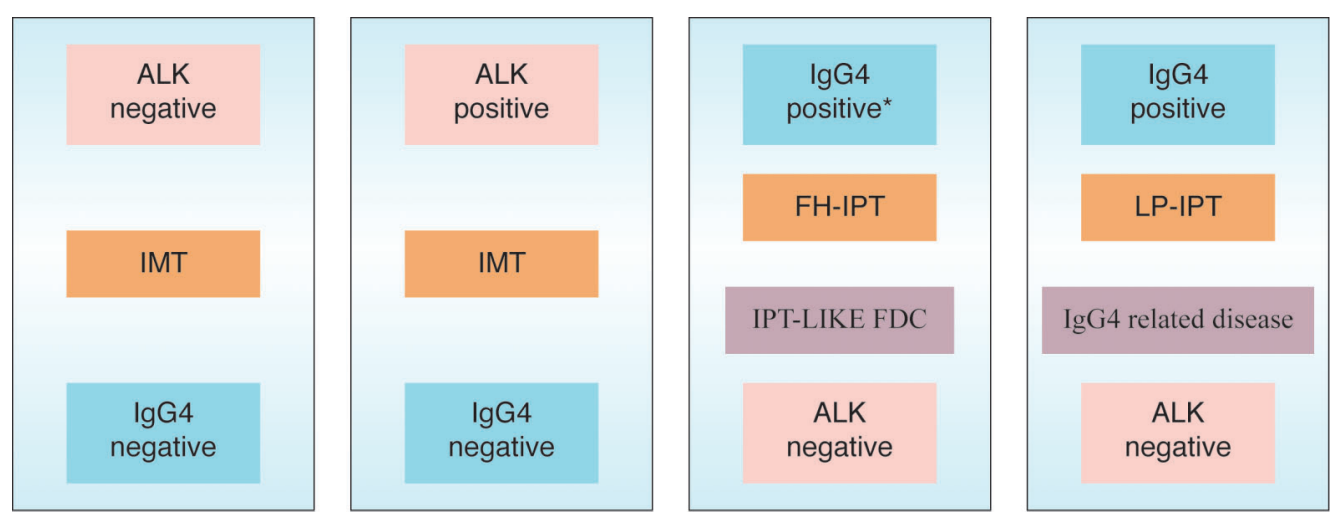

Fig. 5. Although IMT formerly diagnosed as IPT, the expression of ALK and lack of IgG4 demonstrate that IMT is a distinctive neoplasm and should be separated from other lesions that are included in the broad category of IPT.

the rare incidence of metastasis. Recently, there has been a search for prognostic factors to guide treatment and predict outcome. Evidence indicates that more aggressive IMT are associated with cellular atypia, ganglion-like cells, p53 expression, and aneuploidy. ${ }^{21}$ However, these features were not conclusive in a recent series by Coffin et al. ${ }^{22}$ They observed that recurrence was associated with an abdominopelvic site, larger size, and older age of the patient. On the other hand, metastases were more frequently observed in younger patients. They also found that ALK reactivity may be a favorable prognostic factor because all metastatic IMTs in the series were non-reactive to ALK. In IMT of the liver, a few cases with local recurrence and an aggressive clinical course have been described. ${ }^{24}$ For this reason, it is not possible to delineate the prognostic parameters that predict aggressive behavior of IMT of the liver.

Management of IMT is controversial. Some authors have advocated a conservative approach with the diagnosis of IMT. ${ }^{8}$ However, the natural course of these tumors without intervention is not fully known. Importantly, when recurrences occur, it is usually in cases with incomplete resection and no chemotherapy or radiotherapy was provided. Therefore, it is suggested that surgical resection should be recommended for all lesions if not prohibited by anatomic location or morbidity. ${ }^{9,10}$

Recently, it has been proposed that in cases of an unresectable and metastatic ALK-expressing IMT, ALK inhibitors may provide therapeutic benefit and durable responses may be achieved. ${ }^{38}$ However, the data is very limited, because of the rarity and indolent course of IMT.

\section{Conclusions}

IMT is a distinctive, myofibroblastic neoplasm that shows a tendency for local recurrence and metastasis. According to literature, IMT of the liver is exceedingly rare, and it can mimic malignant tumors. There are no known unique diagnostic clinical, laboratory, or radiological features, and the definitive diagnosis of IMT of the liver relies on pathological evaluation. Chromosomal translocations leading to activation of the ALK can be detected in IMT of the liver, and immunohistochemistry for ALK expression in IMT reliably predicts the presence of an ALK gene rearrangement. However, its prognostic significance remains to be elucidated. IMT of the liver is different from IgG4-related hepatic diseases in terms of the ALK expression, the low level of IgG4 positive cell infiltration, and the lack of obstructive phlebitis. Management of IMT of the liver is controversial. However, it has been suggested that surgical resection should be recommended for all lesions if contraindicated. Finally, the rarity of IMT in the liver and the lack of diagnostic signs and symptoms should not prevent consideration in the differential diagnosis of liver tumors, especially in patients with tumor markers in normal range.

\section{Conflict of interest}

None

\section{Author contributions}

Analyzing the data and writing the paper (GÖE).

\section{References}

[1] Coffin CM, Watterson J, Priest JR, Dehner LP. Extrapulmonary inflammatory myofibroblastic tumor (inflammatory pseudotumor). A clinicopathologic and immunohistochemical study of 84 cases. Am J Surg Pathol 1995;19:859872.

[2] Cook JR, Dehner LP, Collins MH, Ma Z, Morris SW, Coffin CM, et al. Anaplastic lymphoma kinase (ALK) expression in the inflammatory myofibroblastic tumor: a comparative immunohistochemical study. Am J Surg Pathol 2001, 25:1364-1371.

[3] Coffin CM, Patel A, Perkins S, Elenitoba-Johnson KS, Perlman E, Griffin CA, et al. ALK1 and p80 expression and chromosomal rearrangements involving 2 p23 in inflammatory myofibroblastic tumor. Mod Pathol 2001;14:569-576.

[4] Coffin CM, Fletcher JA. Inflammatory myofibroblastic tumour. In: Fletcher CDM, Unni K, Mertens F, editors. Pathology and genetics of tumours of soft tissue and bone. World Health Organization classification of tumours. Lyon: IARC Press; 2002.

[5] Pack GT, Baker HW. Total right hepatic lobectomy; report of a case. Ann Surg 1953;138:253-258.

[6] Levy S, Sauvanet A, Diebold M, Marcus C, Da Costa N, Thiefin G. Spontaneous regression of an inflammatory pseudotumour of the liver presenting as an obstructing malignant biliary tumour. Gastrointest Endosc 2001;53:371-374.

[7] Torzilli G, Inoue K, Midorikawa Y, Hui AM, Takayama T, Makuuchi M. Inflammatory pseudotumours of the liver: prevalence and clinical impact in surgical patients. Hepatogastroenterology 2001;48:1118-1123.

[8] Golsmith PJ, Loganathan A, Jacob M, Ahmad N, Toogood G], Lodge JP, et al. Inflammatory pseudotumors of the liver: a spectrum of presentation and management options. Eur J Surg Oncol 2009:35:1295-1298.

[9] Tang L, Lai ECH, Cong W, Li A, Fu S, Pan Z, et al. Inflammatory myofibroblastic tumor of the liver: a cohort study. World J Surg 2010;34: 309-313. 
[10] Nagarajan S, Jayabose S, McBride W, Prasadh I, Tanjavur V, Marvin MR, et al. Inflammatory myofibroblastic tumor of the liver in children: case report and review of the literature. J Pediatr Gastroenterol Nutr 2013;57:277-280.

[11] Gleason BC, Hornick JL. Inflammatory myofibroblastic tumours: where are we now? J Clin Pathol 2008;61:428-437.

[12] Ogawa T, Yokoi H, Kawarada Y. A case of inflammatory pseudotumor of the liver causing elevated serum CA19-9 levels. Am J Gastroenterol 1998;93: 2551-2555.

[13] Deng FT, Li YX, Ye L, Tong L, Yang XP, Chai XQ. Hilar inflammatory pseudotumor mimicking hilar. Cholangiocarcinoma. Hepatobiliary Pancreat Dis Int 2010;9:219-221.

[14] Teranishi N, Yoshida H, Mamada $Y$, Taniai N, Mizuguchi $Y$, Shimizu T, et al. Inflammatory pseudotumor in the Spiegel lobe of the liver of an elderly woman. J Nippon Med Sch 2005;72:121-126.

[15] Ueda M, Yukihide Y, Ogawa K, Haga H, Ogura Y, Ito T, et al. A case of inflammatory pseudotumor of the liver hilum successfully treated with aggressive hepatectomy. J Pediatr Surg 2003;38:E9-E11.

[16] Hoosein MM, Tapuria N, Standish RA, Koti RS, Webster G], Millar AD, et al. Inflammatory pseudotumour of the liver: the residuum of a biliary cystadenoma? Eur J Gastroenterol Hepatol 2007;19:333-336.

[17] Horiuchi R, Uchida T, Kojima T, Shikata T. Inflammatory pseudotumor of the liver. Clinicopathologic study and review of the literature. Cancer 1990;65: 1583-1590.

[18] Tambay R, Cote J, Bourgault AM, Villeneuve JP. An unusual case of hepatic abscess. Can J Gastroenterol 2001;15:615-617.

[19] Kim HS, Park NH, Park KA, Kang SB. A case of pelvic actinomycosis with hepatic actinomycotic pseudotumor. Gynecol Obstet Invest. 2007;64:95-99.

[20] Faraj W, Ajouz H, Mukherji D, Kealy G, Shamseddine A, Khalife M. Inflammatory pseudo-tumor of the liver: a rare pathological entity. World J Surg Oncol 2011;9:5.

[21] Hussong JW, Brown M, Perkins SL, Dehner LP, Coffin CM. Comparison of DNA ploidy, histologic, and immunohistochemical findings with clinical outcome in inflammatory myofibroblastic tumors. Mod Pathol 1999;12:279-286.

[22] Coffin CM, Hornick JL, Fletcher CD. Inflammatory myofibroblastic tumor: comparison of clinicopathologic, histologic, and immunohistochemical features including ALK expression in atypical and aggressive cases. Am J Surg Pathol 2007;31:509-520.

[23] Donner LR, Trompler RA, White RRt. Progression of inflammatory myofibroblastic tumor (inflammatory pseudotumor) of soft tissue into sarcoma after several recurrences. Hum Pathol 1996;27:1095-1098.

[24] Chen ST, Lee JC MD. An inflammatory myofibroblastic tumor in liver with ALK and RANBP2 gene rearrangement: combination of distinct morphologic, immunohistochemical, and genetic features. Hum Pathol 2008;39:18541858

[25] Griffin CA, Hawkins AL, Dvorak C, Henkle C, Ellingham T, Perlman EJ, et al Recurrent involvement of 2 p23 in inflammatory myofibroblastic tumors. Cancer Res 1999;59:2776-2780.

[26] Lawrence B, Perez-Atayde A, Hibbard MK, Rubin BP, Dal Cin P, Pinkus JL, et al. TPM3-ALK and TPM4-ALK oncogenes in inflammatory myofibroblastic tumors. Am J Pathol 2000;157:377-384.

[27] Chan JK, Cheuk W, Shimizu M. Anaplastic lymphoma kinase expression in inflammatory pseudotumors. Am J Surg Pathol 2001;25:761-768.

[28] Sukov WR, Cheville JC, Carlson AW, Shearer BM, Piatigorsky EJ, Grogg KL, et al. Utility of ALK-1 protein expression and ALK rearrangements in distinguishing inflammatory myofibroblastic tumor from malignant spindle cell lesions of the urinary bladder. Mod Pathol 2007;20:592-603.

[29] See TC, Davies SE, Appleton DS, Ng CS. CT and angiographic features of hepatic inflammatory myofibroblastic tumour. Clin Radiol 2005;60:718-722.

[30] Solomon G], Kinkhabwala MM, Akhtar M. Inflammatory myofibroblastic tumor of the liver. Arch Pathol Lab Med 2006;130:1548-1551.

[31] Sürer S, Bozova S, Gökhan GA, Elpek GO. Inflammatory myofibroblastic tumor of the liver.a case report. Turk J Gastroenterol 2009;20:129-134.

[32] Yamamoto $H$, Yamaguchi $H$, Aishima S, Oda $Y$, Kohashi $K$, Oshiro $Y$, et al. Inflammatory myofibroblastic tumor versus IgG4-related sclerosing disease and inflammatory pseudotumor a comparative clinicopathologic study. Am J Surg Pathol 2009;33:1330-1340.

[33] Salakos C, Nikolakopoulou NM, De Verney Y, Tsamandas AC, Ziambaras T, Petsas $\mathrm{T}$, et al. Anaplastic lymphoma kinase (ALK) positive inflammatory pseudotumor of the liver: conservative treatment long term follw-up. Eur J Pediatr Surg 2010;20:278-280.

[34] Ma Z, Hill DA, Collins MH, , Morris SW, Sumegi J, Zhou M, et al. Fusion of ALK to the Ran-binding protein 2 (RANBP2) gene in inflammatory myofibroblastic tumor. Genes Chromosomes Cancer 2003;37:98-105.

[35] Miettinen M, Sobin LH, Sarlomo-Rikala M. Immunohistochemical spectrum of GISTs at different sites and their differential diagnosis with a reference to CD117 (KIT). Mod Pathol 2000;13:1134-1142.

[36] Nascimento AF, Ruiz R, Hornick JL, Fletcher CD. Calcifying fibrous 'pseudotumor': clinicopathologic study of 15 cases and analysis of its relationship to inflammatory myofibroblastic tumor. Int J Surg Pathol 2002;10:189-196.

[37] Zen Y, Fujii T, Sato Y, Masuda S, Nakanuma Y. Pathological classification of hepatic inflammatory pseudotumor with respect to IgG4-related disease. Mod Pathol 2007;20:884-894.

[38] Tothova Z, Wagner AJ. Anaplastic lymphoma kinase-directed therapy in inflammatory myofibroblastic tumors. Curr Opin Oncol 2012;24:409-413. 